AIAA Journal, Vol. 36, No. 3, March 1998, pp. 491-493.

\title{
The Direct Updating of Damping and Stiffness Matrices
}

\author{
M. I. Friswell \\ University of Wales Swansea, Swansea SA2 8PP, UK \\ D. J. Inman and D. F. Pilkey \\ Virginia Polytechnic Institute and State University, Blacksburg, VA 24061-0219
}

\section{Introduction}

Model updating is becoming a common method to improve the correlation between finite element models and measured data ${ }^{1,2}$. A number of approaches to the problem exist, based on the type of parameters that are updated and the measured data that is used. This paper concentrates on a direct updating method based on measured modal data. These methods update complete structural matrices, so that the updated matrices are those closest to the initial analytical matrices, but reproduce the measured data. For example, Baruch $^{3,4}$ considered the mass matrix to be exact and updated the stiffness matrix. A preliminary step estimated the mass normalised eigenvectors closest to the measured eigenvectors. Berman ${ }^{5,6}$ questioned whether the mass matrix should be considered exact, and updated both the mass matrix and the stiffness matrix. Baruch ${ }^{7}$ described these methods as reference basis methods, since one of three quantities (the measured modal data, and the analytical mass and stiffness matrices) is assumed to be exact, or the reference, and the other two are updated. Caesar ${ }^{8}$ extended this approach and produced a range of methods based on optimising a number of cost functions. All the methods described thus far share the feature that only one quantity is updated at a time. Wei ${ }^{9-11}$ updated the mass and stiffness matrices simultaneously, using the measured modal data as a reference. The constraints imposed were mass orthogonality, the equation of motion and the symmetry of the updated matrices.

All of the methods described above used real mode shapes and natural frequencies. The measured mode shapes were processed to produce the equivalent real modes. This paper extends the reference basis methods by updating the damping matrix. Fuh et al. ${ }^{12}$ proposed a method that updates the mass and damping matrices with the constraint of orthogonality. The stiffness matrix is then updated to ensure the measured modal model is reproduced. Weighted norms between the initial and updated mass, damping and stiffness matrices are minimised. In this paper the mass matrix is assumed correct and the damping and stiffness matrices are updated simultaneously, so that the updated model reproduces the measured modal data. 


\section{The Updating Method}

Following the method of Baruch ${ }^{3,4}$, who updated the stiffness matrix only, the difference between the initial and updated damping and stiffness matrices is minimised, with the constraints that the eigenvalue equation is satisfied and that the damping and stiffness matrices are symmetric (and of course real). Thus, the penalty function to be minimised is given by,

$$
J=\frac{1}{2}\left\|\mathbf{N}^{-1}\left[\mathbf{K}-\mathbf{K}_{a}\right] \mathbf{N}^{-1}\right\|^{2}+\frac{1}{2} \mu\left\|\mathbf{N}^{-1}\left[\mathbf{C}-\mathbf{C}_{a}\right] \mathbf{N}^{-1}\right\|^{2}
$$

subject to

$$
\begin{gathered}
\mathbf{M}_{a} \Phi \Lambda^{2}+\mathbf{C} \Phi \Lambda+\mathbf{K} \Phi=\mathbf{0} \\
\mathbf{C}=\mathbf{C}^{T} \quad \mathbf{K}=\mathbf{K}^{T}
\end{gathered}
$$

where $\mathbf{N}=\mathbf{M}_{a}{ }^{1 / 2}, \mathbf{M}_{a}, \mathbf{C}_{a}$ and $\mathbf{K}_{a}$ are the initial, analytical mass, damping and stiffness matrices, $\mathbf{C}$ and $\mathbf{K}$ are the updated damping and stiffness matrices, and $\Phi$ and $\Lambda$ are the measured eigenvector and eigenvalue matrices. The extension for other weighting matrices, $\mathbf{N}$, and indeed for different weighting of the damping and stiffness matrices, is straightforward. A full set of modes are not measured, so $\Phi$ is not square, but all degrees of freedom (DoFs) are assumed measured. If only a subset of the DoFs is measured then the model must be reduced or the mode shapes expanded. $\Lambda$ is a diagonal matrix with the measured eigenvalues on the diagonal. The parameter $\mu$ in Equation (1) is to enable the damping and stiffness terms to be weighted. Often the magnitude of the stiffness terms are far greater than the damping terms, and so if $\mu$ was not present more weight would be given to the stiffness terms, leading to a poor estimate of the damping matrix. The value of $\mu$ is selected based on 'experience', often using the results from a range of values. This will be discussed further in the example. Note that $\mathrm{Wei}^{9-11}$ produced a similar method for updating mass and stiffness matrices simultaneously, although he didn't include a weighting factor similar to $\mu$. Including such a factor does mean that closed form solutions for the updated matrices are unlikely, but in practice including this weighting is vital.

The Lagrange multiplier method will now be used to solve the optimisation problem. The augmented penalty function based on Equation (1) and the constraints is 


$$
\begin{gathered}
J=\frac{1}{2}\left\|\mathbf{N}^{-1}\left[\mathbf{K}-\mathbf{K}_{a}\right] \mathbf{N}^{-1}\right\|^{2}+\frac{1}{2} \mu\left\|\mathbf{N}^{-1}\left[\mathbf{C}-\mathbf{C}_{a}\right] \mathbf{N}^{-1}\right\|^{2}+ \\
\sum_{i, j=1}^{n} \gamma_{K i j}\left(k_{i j}-k_{j i}\right)+\sum_{i, j=1}^{n} \gamma_{C i j}\left(c_{i j}-c_{j i}\right)+ \\
2 \sum_{i=1}^{n} \sum_{j=1}^{m} \gamma_{\Lambda i j} \sum_{h=1}^{n}\left(k_{i h} \phi_{h j}+c_{i h} \phi_{h j} \lambda_{j}+m_{i h} \phi_{h j} \lambda_{j}^{2}\right)+ \\
2 \sum_{i=1}^{n} \sum_{j=1}^{m} \bar{\gamma}_{\Lambda i j} \sum_{h=1}^{n}\left(k_{i h} \bar{\phi}_{h j}+c_{i h} \bar{\phi}_{h j} \bar{\lambda}_{j}+m_{i h} \bar{\phi}_{h j} \bar{\lambda}_{j}^{2}\right)
\end{gathered}
$$

where $k_{i j}$ is the $(i, j)$ element of $\mathbf{K}$ and similarly for $\mathbf{C}$ and $\Phi, \lambda_{j}$ is the $j$ th eigenvalue (or the $(j, j)$ element of $\Lambda$ ), $n$ is the number of DoFs, and $m$ is the number of measured modes. The overbar denotes the complex conjugate. The third and fourth terms ensure the updated damping and stiffness matrices are symmetric, and the last 2 terms ensure that the eigenvalue equation is satisfied. The Lagrange multipliers may be formed into 3 matrices in obvious notation: $\Gamma_{\mathrm{K}}$ and $\Gamma_{\mathrm{C}}$ which are real and forced to be skewsymmetric (since otherwise they would not be unique) and $\Gamma_{\Lambda}$ which is complex. Because the eigenvalues and eigenvectors are complex, so must the corresponding Lagrange multiplier, $\Gamma_{\Lambda}$, and the last term in Equation (4) is the complex conjugate of the penultimate term to ensure that $J$ is real.

Equation (4) may be optimised by differentiating $J$ with respect to each element of $\mathbf{K}$ and $\mathbf{C}$, and assembling the results into matrix form. The differentiation with respect to the stiffness gives the following equation,

$$
\mathbf{M}_{a}^{-1}\left[\mathbf{K}-\mathbf{K}_{a}\right] \mathbf{M}_{a}^{-1}+2 \Gamma_{\mathrm{K}}+2 \Gamma_{\Lambda} \Phi^{T}+2 \bar{\Gamma}_{\Lambda} \bar{\Phi}^{T}=\mathbf{0}
$$

Similarly differentiating with respect to the damping matrix gives,

$$
\mu \mathbf{M}_{a}^{-1}\left[\mathbf{C}-\mathbf{C}_{a}\right] \mathbf{M}_{a}^{-1}+2 \Gamma_{\mathrm{C}}+2 \Gamma_{\Lambda} \Lambda \Phi^{T}+2 \bar{\Gamma}_{\Lambda} \bar{\Lambda} \bar{\Phi}^{T}=\mathbf{0}
$$

Using the skew symmetry of $\Gamma_{\mathrm{K}}$, and the symmetry of the mass and stiffness matrices, we can eliminate $\Gamma_{\mathrm{K}}$ from Equation (5) to give,

$$
\mathbf{K}=\mathbf{K}_{a}-\mathbf{M}_{a}\left[\Gamma_{\Lambda} \Phi^{T}+\Phi \Gamma_{\Lambda}^{T}+\bar{\Gamma}_{\Lambda} \bar{\Phi}^{T}+\bar{\Phi} \bar{\Gamma}_{\Lambda}^{T}\right] \mathbf{M}_{a}
$$

Similarly for the damping matrix, from Equation (6),

$$
\mathbf{C}=\mathbf{C}_{a}-\frac{1}{\mu} \mathbf{M}_{a}\left[\Gamma_{\Lambda} \Lambda \Phi^{T}+\Phi \Lambda \Gamma_{\Lambda}^{T}+\bar{\Gamma}_{\Lambda} \bar{\Lambda} \bar{\Phi}^{T}+\bar{\Phi} \bar{\Lambda} \bar{\Gamma}_{\Lambda}^{T}\right] \mathbf{M}_{a}
$$


If we knew the Lagrange multiplier matrix $\Gamma_{\Lambda}$ we could calculate the updated damping and stiffness matrices from Equations (7) and (8). We can obtain a set of equations for this Lagrange multiplier matrix by combining Equations (7) and (8) with the constraint, Equation (2), to give,

$$
\begin{aligned}
& \mathbf{M}_{a}\left[\Gamma_{\Lambda} \Phi^{T}+\Phi \Gamma_{\Lambda}^{T}+\bar{\Gamma}_{\Lambda} \bar{\Phi}^{T}+\bar{\Phi} \bar{\Gamma}_{\Lambda}^{T}\right] \mathbf{M}_{a} \Phi+ \\
& \frac{1}{\mu} \mathbf{M}_{a}\left[\Gamma_{\Lambda} \Lambda \Phi^{T}+\Phi \Lambda \Gamma_{\Lambda}^{T}+\bar{\Gamma}_{\Lambda} \bar{\Lambda} \bar{\Phi}^{T}+\bar{\Phi} \bar{\Lambda} \bar{\Gamma}_{\Lambda}^{T}\right] \mathbf{M}_{a} \Phi \Lambda= \\
& \mathbf{M}_{a} \Phi \Lambda^{2}+\mathbf{C}_{a} \Phi \Lambda+\mathbf{K}_{a} \Phi
\end{aligned}
$$

Equation (9) is a set of $2 \mathrm{~nm}$ linear equations (real and imaginary parts) for the $\mathrm{nm}$ complex elements of the matrix $\Gamma_{\Lambda}$. Baruch ${ }^{3,4}$ and $\mathrm{Wei}^{9-11}$ simplified these equations and gave closed form solutions, but the presence of the factor $\mu$ makes this impossible here. Also the eigenvector normalisation for complex modes is not so straight forward as using mass normalised real eigenvectors. Even so, Equation (9) should be sufficient to obtain $\Gamma_{\Lambda}$ and then the updated damping and stiffness matrices may be obtained from Equations (7) and (8). In practice, the solution to equation (9) may be ill-conditioned, in which case a pseudo inverse must be applied based on a singular value decomposition.

\section{A Numerical Example}

The algorithm outlined in this paper will be tested on a simulated example of a steel, 10 element cantilever beam, of length $1 \mathrm{~m}$, breadth $25 \mathrm{~mm}$ and thickness $50 \mathrm{~mm}$. The initial, analytical model has no damping, and Guyan reduction ${ }^{13}$ is used to reduce the analytical model to the 10 translational DoFs. The 'measured' eigenvalues and eigenvectors are derived from a 'damaged' beam model. The damage is modelled as a reduction in the stiffness of element 4 by $25 \%$. The damping in element 4 is also increased, and is assumed to be $10^{-5}$ times the stiffness of element 4 . This damage model is motivated by the realisation that damage often reduces stiffness and adds damping locally. The full 20 DoF model, including rotational DoFs is used to calculate the 'measured' data. Only the translational DoFs and the 5 lowest frequency modes are assumed to be measured.

The proposed algorithm was applied for a wide range of weighting values, $\mu$. Figure 1 shows the effect of changing $\mu$ on the weighted norms of the change in the damping and stiffness matrices. The changes are weighted by the analytical mass matrix and represent the two terms in the penalty function, Equation (1). As $\mu$ is increased the change in the damping matrix becomes smaller and the change in the stiffness matrix becomes larger. As expected this change in both norms is monotonic. Often in regularisation type applications, with which this approach has some similarities, the equivalent plot has an ' $L$ ' shape and an optimum value of $\mu$ may be obtained. The difference here is that the weight given to different parts of the updated model are changed, rather than the weight 
between the measured and analytical data. Even so, a good compromise value of $\mu$ would be one where the norms change considerably. The starred value in Figure $1(\mu=60)$ would be a good compromise.

The measured eigenvalues are not reproduced exactly because a pseudo inverse was required to calculate the Lagrange multiplier matrix. There are also two potentially serious problems that arise in the method. The first problem is that the matrices are not guaranteed to be positive definite, and for the damping matrices this means that some of the higher modes can have negative damping (that is be unstable). Extra low frequency modes can also be introduced. Furthermore the connectivity of the original finite element model is not necessarily preserved. The connectivity problem has been addressed in the standard methods by Kabé ${ }^{14}$ and Smith and Beattie ${ }^{15}$.

\section{Conclusions}

This note has outlined an extension to the available direct methods of model updating to estimate both the damping and stiffness matrices of a structure. The method minimises the change in the damping and stiffness matrices, with the constraint that the measured modal data is reproduced. The approach was demonstrated using a simulated example, and produced reasonable results. The algorithm does not guarantee that the updated matrices will be positive definite and the connectivity of the original finite element model is not necessarily preserved. Also extra modes may be introduced in the frequency range of interest. These problems have been addressed in the standard methods, and are the subject of further investigation.

\section{Acknowledgements}

Dr. Friswell gratefully acknowledges the support of the EPSRC through the award of an Advanced Fellowship.

\section{References}

1. Friswell, M.I. and Mottershead, J.E., Finite Element Model Updating in Structural Dynamics, Kluwer Academic Publishers, 1995.

2. Mottershead, J.E. and Friswell, M.I., "Model Updating in Structural Dynamics: A Survey," Journal of Sound and Vibration, Vol. 167, No. 2, 1993, pp. 347-375.

3. Baruch, M., "Optimization Procedure to Correct Stiffness and Flexibility Matrices using Vibration Data," AIAA Journal, Vol. 16, No. 11, 1978, pp. 1208-1210.

4. Baruch, M. and Bar-Itzack, I.Y., "Optimal Weighted Othogonalization of Measured Modes," AIAA Journal, Vol. 16, No. 4, 1978, pp. 346-351.

5. Berman, A., "Comment on 'Optimal Weighted Othogonalization of Measured Modes'," AIAA Journal, Vol. 17, No. 8, 1979, pp. 927-928.

6. Berman, A. and Nagy, E.J., "Improvement of a Large Analytical Model using Test Data," AIAA Journal, Vol. 21, No. 8, 1983, pp. 1168-1173. 
7. Baruch, M., "Methods of Reference Basis for Identification of Linear Dynamic Structures," AIAA Paper No. 82-0769, 23rd Structures, Structural Dynamics and Materials Conference, Part 2, New Orleans, Louisiana, 1982, pp. 557-563.

8. Caesar, B., "Update and Identification of Dynamic Mathematical Models," 4th IMAC, Los Angeles, California, 1986, pp. 394-401.

9. Wei, F.-S., "Structural Dynamic Model Identification using Vibration Test Data," $7^{\text {th }}$ IMAC, Las Vegas, Nevada, 1989, pp. 562-567.

10. Wei, F.-S., "Structural Dynamic Model Improvement using Vibration Test Data," AIAA Journal, Vol. 28, No. 1, 1990, pp. 175-177.

11. Wei, F.-S., "Mass and Stiffness Interaction Effects in Analytical Model Modification," AIAA Journal, Vol. 28, No. 9, 1990, pp. 1686-1688.

12. Fuh, J-S., Chen, S-Y. and Berman, A., "System Identification of Analytical Models of Damped Structures," AIAA/ASME/ASCE/AHS 25th Structures, Structural Dynamics \& Materials Conference, Palm Springs, California, 1984, Paper No. AIAA-84-0926.

13. Guyan, R.J., "Reduction of Stiffness and Mass Matrices," AIAA Journal, Vol. 3, No. 2, 1965, p. 380.

14. Kabé, A.M., "Stiffness Matrix Adjustment using Modal Data," AIAA Journal, Vol. 23, No. 9, 1985, pp. 1431-1436.

15. Smith, S.W. and Beattie, C.A., "Secant-Method Adjustment for Structural Models," AIAA Journal, Vol. 29, No. 1, 1991, pp. 119-126.

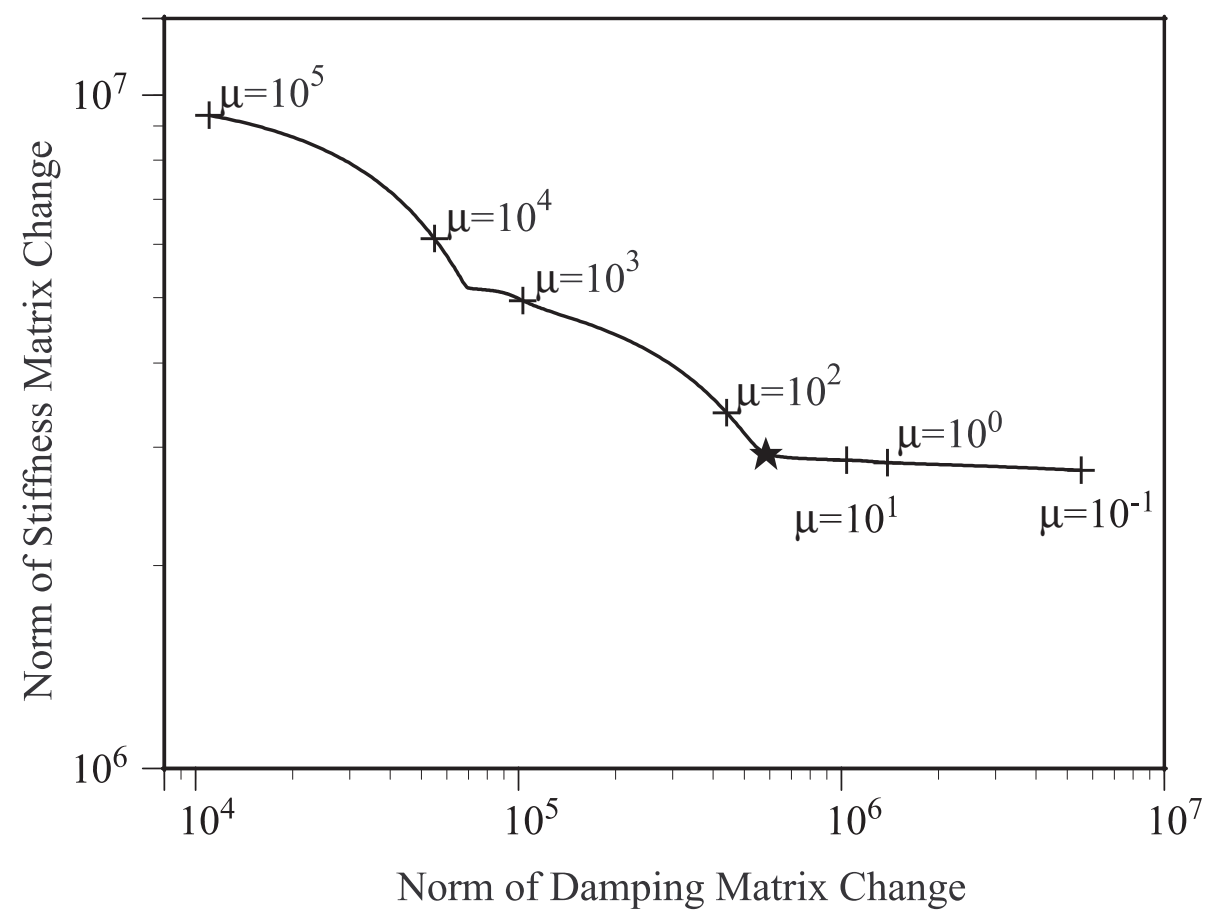

Figure 1.The Variation of the Norms of the Changes in the Damping and Stiffness Matrices with the Weighting Factor 